\title{
Comparison of methylation patterns generated from genomic and cell-line derived DNA using the Illumina Infinium MethylationEPIC BeadChip array
}

\author{
L. J. Smyth*, J. Kilner, A. P. Maxwell and A. J. McKnight
}

\begin{abstract}
Objectives: Genomic DNA (gDNA) is the optimal source of DNA for methylation analysis. This study compared methylation patterns in gDNA derived from blood with cell-line derived DNA (cIDNA) from the same individuals. The cIDNA had been generated via an Epstein-Barr virus transformation of the participant's lymphocytes. This analysis sought to determine whether cIDNA has the potential to be utilised in lieu of finite/unavailable gDNA in methylation analyses using Illumina Infinium MethylationEPIC BeadChip arrays that assess 862,927 CpG sites.

Results: DNA samples were divided into two groups with eight gDNA and eight matched cIDNA samples compared in each group ( $n=16$ individuals with 32 samples in total). Methylation patterns for gDNA samples generated for both groups were compared to the clDNA equivalent samples using Parte ${ }^{\circledR}$ Genomics Suite ${ }^{\circledR}$ to assess whether the significantly different CpG sites were consistent between both groups. In total, 28,632 CpG sites with significantly different levels of methylation $\left(p<\times 10^{-8}\right)$ were common to both groups while $828,072 \mathrm{CpG}$ sites assessed by the MethylationEPIC array were not significantly different in either group. This indicates that there is potential for cIDNA to be used as a replacement for finite gDNA samples when absolutely necessary in DNA methylation studies.
\end{abstract}

Keywords: Cell-line, Diabetes, DNA, Epigenetics, Genomic, Methylation, MethylationEPIC

\section{Introduction}

DNA methylation is a key epigenetic feature, defined as the covalent addition of a methyl group to the 5-carbon position of a cytosine nucleotide at cytosine-phosphateguanine $(\mathrm{CpG})$ sites. These $\mathrm{CpG}$ sites frequently cluster within $\mathrm{CpG}$ islands that are repetitive sequences often located near gene promoters. DNA methylation has been associated with several complex diseases including chronic kidney disease (CKD) and diabetes [1-9].

The current gold-standard method of assessing DNA methylation patterns is through whole-genome

*Correspondence: laura.smyth@qub.ac.uk

Molecular Epidemiology Research Group, Centre for Public Health,

Queen's University of Belfast, Belfast, UK bisulphite sequencing (WGBS), a method which provides single-nucleotide resolution and whole-genome coverage of approximately $95 \%$ of all CpG sites. However, this method requires large quantities of input DNA [10] and is financially prohibitive for many large-scale research studies. Illumina's Infinium methylation arrays provide a user-friendly, cost-effective alternative, which require a lower input concentration of DNA $[11,12]$. The Infinium MethylationEPIC array contains the most modern available technology and provides coverage of $862,927 \mathrm{CpG}$ sites [13].

The aim of this study was to compare blood-derived genomic DNA (gDNA) and DNA derived from EpsteinBarr virus (EBV) transformed cell-lines (clDNA) from the same participants using data generated using Illumina's 
Infinium MethylationEPIC BeadChip array. This provided an opportunity to evaluate methylation data generated from the more readily available clDNA samples compared to gDNA samples.

\section{Main text \\ Methods \\ Sample cohort}

All participants were of White ancestry from the British Isles and provided written informed consent for research. Each participant was recruited as part of the All IrelandWarren 3-Genetics of Kidneys in Diabetes (GoKinD) UK Collection. DNA was frozen in multiple aliquots having been extracted from whole blood using the salting out method and normalised following PicoGreen quantitation. EBV transformation of participants' peripheral blood leukocytes was performed by the European Collection of Authenticated Cell Cultures (ECACC) to create clDNA [14].

This study was conducted on 16 participants (with both gDNA and clDNA available for analyses). Eight participants were individuals with $\geq 10$ years duration of type 1 diabetes (T1D) who had also been diagnosed with diabetic kidney disease (T1DKD) defined as persistent macroalbuminuria $(\geq 500 \mathrm{mg} / 24 \mathrm{~h}$ ), estimated glomerular filtration rate $(\mathrm{eGFR})<60 \mathrm{~mL} / \mathrm{min} / \mathrm{m}^{2}$ and hypertension (blood pressure $\geq 135 / 85 \mathrm{mmHg}$ ). The remaining eight individuals had $\geq 15$ years duration of T1D and no evidence of renal disease on repeat testing (eGFR $>60 \mathrm{~mL} /$ $\mathrm{min} / \mathrm{m}^{2}$ ). Duration of diabetes differed by $\leq 2$ years and age at diagnosis $\leq 5$ years. Participants were divided into two groups of eight and the overall characteristics are included within Table 1. Both gDNA and clDNA samples were analysed for all included individuals. Each case and control gDNA sample was compared to the matched clDNA sample generated from blood taken from the same individual.

\section{Infinium MethylationEPIC BeadChip array}

Blood-derived DNA for each individual, both gDNA and clDNA (800 ng), was bisulphite treated (BST) using the EZ DNA Methylation ${ }^{\text {TM }}$ Kit (Zymo Research, USA) using the manufacturer's instructions. All samples were analysed together by the same individual, in the same laboratory.

To assess the methylation status of the CpG sites, the Infinium MethylationEPIC BeadChip array was used following the manufacturer's instructions. This array quantitatively targets 862,927 CpG sites across the genome. Cases and controls were randomly distributed across each array. This high throughput platform evaluated individual methylation levels ( $\beta$ values) for each $\mathrm{CpG}$ site, ranging from 0 for unmethylated to 1 for complete methylation.

\section{Quality control and statistical analyses}

Raw methylation data was assessed for dye bias and quantile normalised as previously reported [1]. Quality control (QC) included evaluation of the bisulphite treatment conversion efficiency, dye specificity, hybridisation, and staining. This was assessed using GenomeStudio v2011 and BeadArray Controls Reporter software platforms (both Illumina).

Table 1 Characteristics of the individuals present within the Infinium MethylationEPIC BeadChip array analysis

\begin{tabular}{|c|c|c|}
\hline Characteristics & Group 1 & Group 2 \\
\hline Number of individuals & $\begin{array}{l}8 \text { (4 gDNA + cIDNA with DKD; } 4 \text { gDNA + cIDNA with } \\
\text { T1D and no renal disease) }\end{array}$ & $\begin{array}{l}8 \text { (4 gDNA + cIDNA with DKD; } 4 \\
\text { gDNA + cIDNA with T1D and no } \\
\text { renal disease) }\end{array}$ \\
\hline Average age of T1D diagnosis & 15 years & 17 years \\
\hline Average duration of diabetes & 35 years & 30 years \\
\hline Females:Males & $5: 3$ & $5: 3$ \\
\hline Average BMI & 27.0 & 24.4 \\
\hline Average BP (mmHg) & 132/79 & $134 / 80$ \\
\hline Hypertension & 4 & 4 \\
\hline Smoking status & $\begin{array}{l}7 \text { Never } \\
1 \text { Ex }\end{array}$ & $\begin{array}{l}6 \text { Never } \\
1 \text { Ex } \\
1 \text { Current }\end{array}$ \\
\hline $\mathrm{eGFR}>60 \mathrm{~mL} / \mathrm{min} / \mathrm{m}^{2}$ & 4 & 4 \\
\hline eGFR 59-15 mL/min/m² [CKD stages 3-4] & 4 & 3 \\
\hline $\mathrm{eGFR}<15 \mathrm{~mL} / \mathrm{min} / \mathrm{m}^{2}$ [ESRD] & 0 & 1 \\
\hline
\end{tabular}


MethylationEPIC analysis was performed using Partek ${ }^{\circledR}$ Genomics Suite ${ }^{\circledR}$ v7.19.1018. Only significant methylation values $\left(\mathrm{p} \leq \times 10^{-8}\right)$ alongside a fold change of \pm 2 , generated in the analysis between gDNA and cIDNA sample groups were included in the comparative analysis. Partek ${ }^{\circledR}$ Genomics Suite ${ }^{\circledR}$ was employed to complete Gene Ontology (GO) analysis and pathway enrichment analysis using the Kyoto Encyclopedia of Genes and Genomes (KEGG) database.

A schematic view of the methods undertaken in this manuscript is provided in Additional file 1: Appendix S1.

\section{Results}

This research note is focused on the comparison of Infinium MethylationEPIC results for gDNA and cIDNA samples, to determine whether cIDNA has potential to be used in methylation array studies, in place of finite samples of gDNA. Each resulting.idat file generated from the iScan was assessed using Illumina's BeadArray Controls Reporter software. This software assessed the data in connection with a pre-set standard set of controls. These QC results are included in Additional file 2: Table S1.

The resulting.idat files were analysed using Genome Studio v2011 and Partek ${ }^{\circledR}$ Genomics Suite ${ }^{\circledR}$ v7.19.1018. The total number of CpG sites examined by the Infinium MethylationEPIC array was 862,927 . No significant difference in intensity levels was detected.

\section{Differential methylation analysis between matched gDNA and cIDNA samples for group 1 and group 2}

Initially, the methylation patterns identified in the gDNA samples were directly compared to the cIDNA equivalent samples within sample group 1 and then independently within sample group 2. Differentially methylated sites within each group were compared to assess whether the significantly different $\mathrm{CpG}$ sites were consistent between the two groups ( $p$ value $\leq \times 10^{-8}$, fold change $\geq \pm 2$ ). In total, 30,566 CpG sites were significantly different between the gDNA and clDNA samples within group 1 ( $\mathrm{n}=8$ vs. $\mathrm{n}=8$, Additional file 2: Table $\mathrm{S} 2$ ), and 32,921 within group 2 ( $\mathrm{n}=8$ vs. $\mathrm{n}=8$, Additional file 2: Table S3).

Furthermore, $>86 \%(28,632)$ of the CpG sites with significantly different levels of methylation were common to both groups. Only 6223 individual CpG sites differed in their level of methylation between groups 1 and 2, these are included in Additional file 2: Table S4. Therefore, 828,072 CpG sites assessed by this methylationEPIC array were not statistically different.

\section{Differential methylation analysis between matched gDNA and cIDNA samples for all samples}

In the second analysis, all gDNA samples from groups 1 and $2(n=16)$ were directly compared to all clDNA samples from groups 1 and $2(n=16)$. These samples were matched $(n=16$ vs. $n=16)$. Overall, $6.2 \%$ of the $\mathrm{CpG}$ sites covered by the array $(53,764)$ were identified as having significantly different levels of methylation between the two groups $\left(p \leq \times 10^{-8}\right.$, fold change $\left.\geq \pm 2\right)$. These results are included within and Additional file 2: Table S5 and an additional breakdown is available within Fig. 1.

In summary, of the 53,764 CpG sites which have shown differential methylation (1438/142,137 (1\%) probe I; $52,326 / 720,790$ (7.3\%) probe II on this array), $89 \%$ were hypermethylated in the gDNA sample group. Of the hypermethylated $\mathrm{CpG}$ sites, $15 \%$ are located within islands (1\%), shelves (6\%) and shores (8\%), and 38\% within gene bodies. In comparison, $22 \%$ of the hypomethylated CpG sites were located within islands (2\%), shelves (8\%) and shores (12\%) and $49 \%$ within gene bodies (Fig. 1). The chromosome location breakdown is similar for both hypermethylated and hypomethylated $\mathrm{CpG}$ sites. Additional file 3: Figure S1a-d are included to illustrate the pattern of average beta values, for each of the experimental groups.

\section{GO and KEGG pathway analyses of differentially methylated genes}

In order to assess the functional significance of the significant DNA methylation alterations between gDNA and clDNA, a GO enrichment analysis was undertaken. This assessed the biological processes, cellular components and molecular functions of the genes within which the top-ranked $\mathrm{CpG}$ sites were located. A total of $54 \mathrm{GO}$ functions were found to have an enrichment score $\geq 10$, alongside $\mathrm{p} \leq \times 10^{-8}$ and these are included within Additional file 2: Table S6 and Fig. 2. The processes with the top enrichment scores included signal transduction, signalling transduction activity, calcium ion binding, cell adhesion and immune system processes.

The KEGG pathway database was searched to identify key pathways linked to the genes where the top-ranked differentially methylated CpG sites were located. Eleven pathways were identified (an enrichment score of $\geq 8$, and $\mathrm{p} \leq \times 10^{-5}$ ) which are included within Additional file 2: Table S7. This analysis has shown that differentially methylated genes are involved in pathways including focal adhesion, protein digestion and vascular and smooth muscle contraction. 
a Altered methylation status of CpG sites

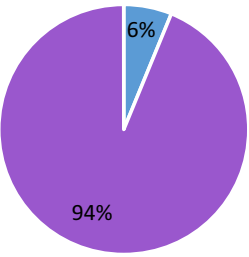

$$
\begin{aligned}
& \text { Altered } \\
& \text { methylation } \\
& \text { between gDNA } \\
& \text { and cIDNA } \\
& \text { No change in } \\
& \text { methylation } \\
& \text { between gDNA } \\
& \text { and cIDNA }
\end{aligned}
$$

b

\section{Percentage of hypermethylated or} hypomethylated $\mathrm{CpG}$ sites

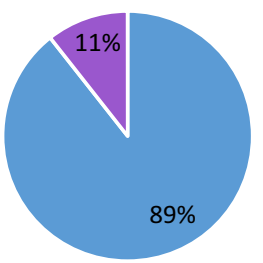

- Hypermethylation in gDNA

- Hypomethylation in gDNA

\section{Hypermethylated CpG sites}

ci Location of hypermethylated CpG sites

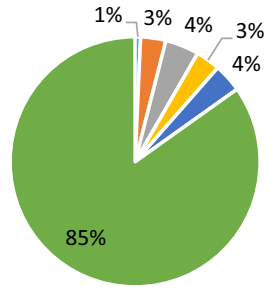

- Island

- N_Shelf

- N_Shore

- S_Shelf

- S_Shore

- Unspecified

The genomic locations of the hypermethylated dmCpGs (Figure 1B) are shown by this chart (Figure $\mathrm{Ci}$ ).

This chart depicts the percentage of

$\mathrm{dmCpGs}$ found by the analysis

undertaken during which gDNA samples

from groups 1 and 2 ( $n=16$ ) were directly

compared to all cIDNA samples from

groups 1 and $2(n=16)$. Only $6 \%$ of $C p G$

sites covered by this array were shown to

be differentially methylated, thus

demonstrating that this EPIC array provides consistent results for $94 \%$ of probes using cIDNA as a viable alternative to the gold standard, but not always available gDNA.

Of the $\mathrm{dmCpGs}$ shown in Figure $1 \mathrm{~A}, 89 \%$ were hypermethylated and $11 \%$ were hypomethylated in the gDNA sample group.

\section{cii Gene structure of hypermethylated} CpG site location

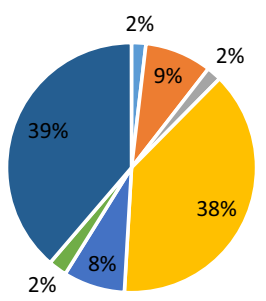

$$
\begin{aligned}
& \text { - 1st Exon } \\
& \text { - 5' UTR } \\
& \text { - 3' UTR } \\
& \text { = Body } \\
& \text { - TSS1500 } \\
& \text { - TSS200 } \\
& \text { - Other }
\end{aligned}
$$

The gene structural locations of the hypermethylated dmCpGs (Figure 1B) are shown by this chart (Figure Cii).

\section{ciii Chromosome location of hypermethylated CpG sites}

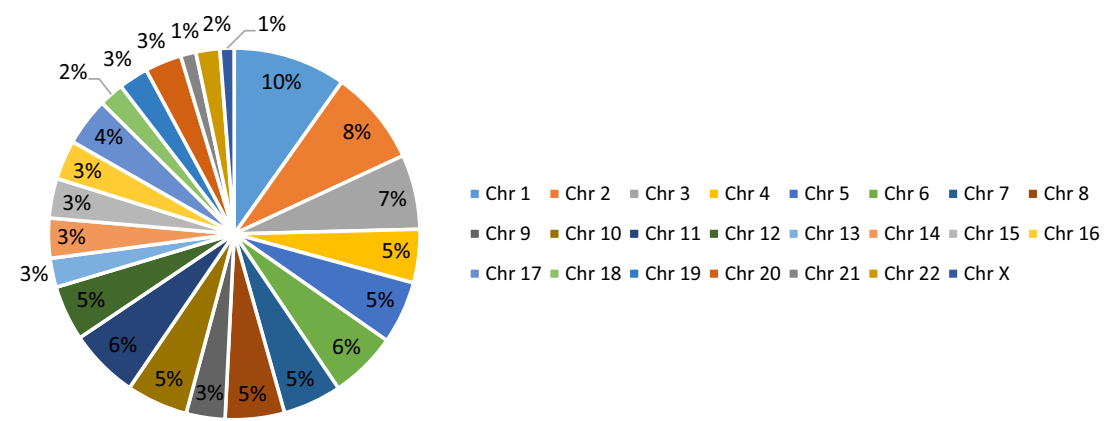

The distribution of the chromosome locations of the hypermethylated dmCpGs (Figure 1B) are illustrated by this chart (Figure Ciii).

Fig. 1 Comparison of differentially methylated CpG sites (dmCpGs) between gDNA and cIDNA. a Altered methylation status of CpG sites between gDNA and cIDNA; $\mathbf{b}$ Percentage of hypermethylated or hypomethylated CpG sites in gDNA; $\mathbf{c}$ Hypermethylated CpG sites; $\mathbf{d}$ Hypomethylated CpG sites; e Percentage of normomethylated, hypermethylated and hypomethylated CpG sites per chromosome. CpG cytosine-phosphate-guanine, cIDNA cell-line DNA, gDNA genomic DNA, N_Shelf North Shelf, N_Shore North Shore, S_Shelf South Shelf, S_Shore South Shore, TSS transcription start site, UTR untranslated region 


\section{d Hypomethylated CpG sites}

di

$$
\begin{aligned}
& \text { Location of hypomethylated CpG } \\
& \text { sites }
\end{aligned}
$$

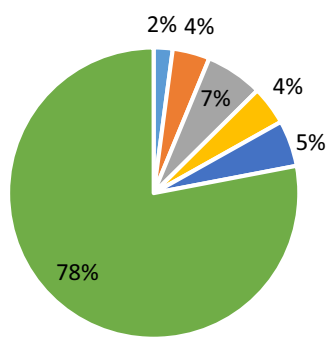

- Island

- N_Shelf

- N_Shore

- S_Shelf

- S_Shore

- Unspecified

The genomic locations of the hypomethylated dmCpGs (Figure 1B) are shown by this chart (Figure $\mathrm{Di}$ ). dii Gene structure of hypomethylated CpG site location

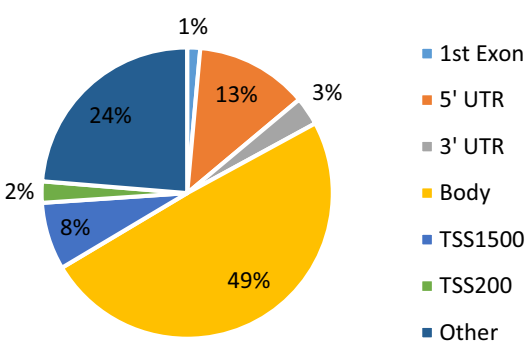

The gene structural locations of the hypomethylated dmCpGs (Figure 1B) are shown by this chart (Figure Dii).

\section{diii Chromosome location of hypomethylated CpG sites}

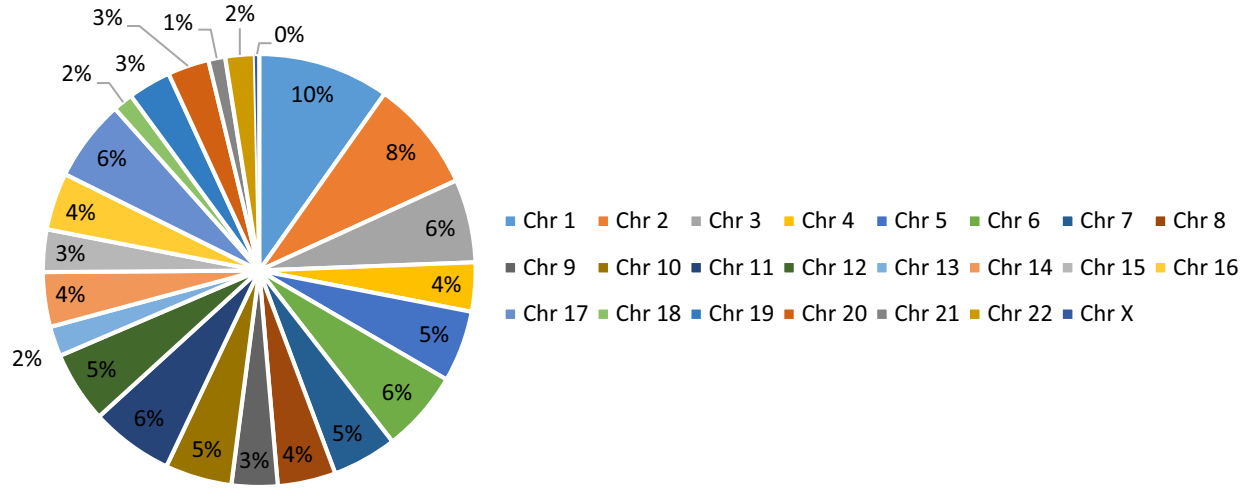

The distribution of the chromosome locations of the hypomethylated dmCpGs (Figure 1B) are illustrated by this chart (Figure Diii).

\section{e Percentage of normomethylated, hypermethylated and hypomethylated $\mathrm{CpG}$ sites per} chromosome.

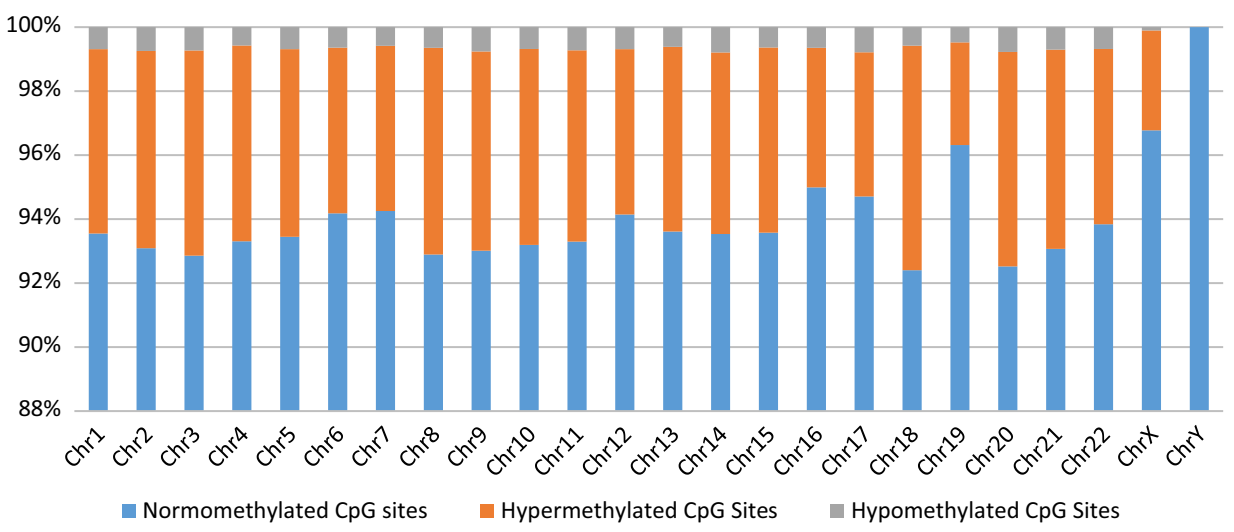

This chart displays the percentage of normo/hyper/hypo methylated $\mathrm{CpG}$ sites, distributed across chromosomes.

Fig. 1 continued 


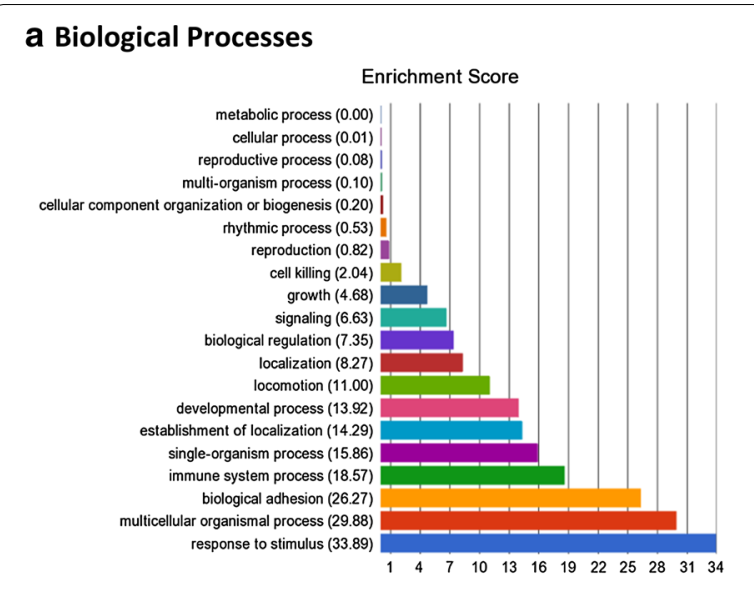

\section{b Cellular Components}

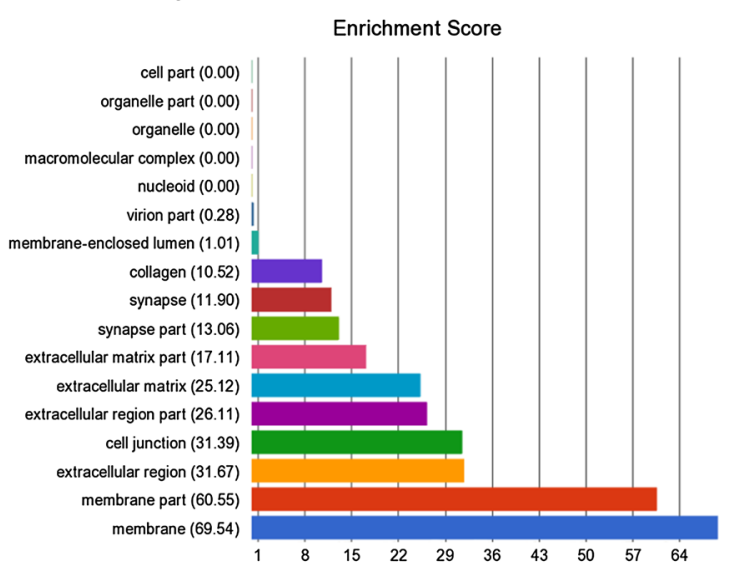

\section{Molecular Function}

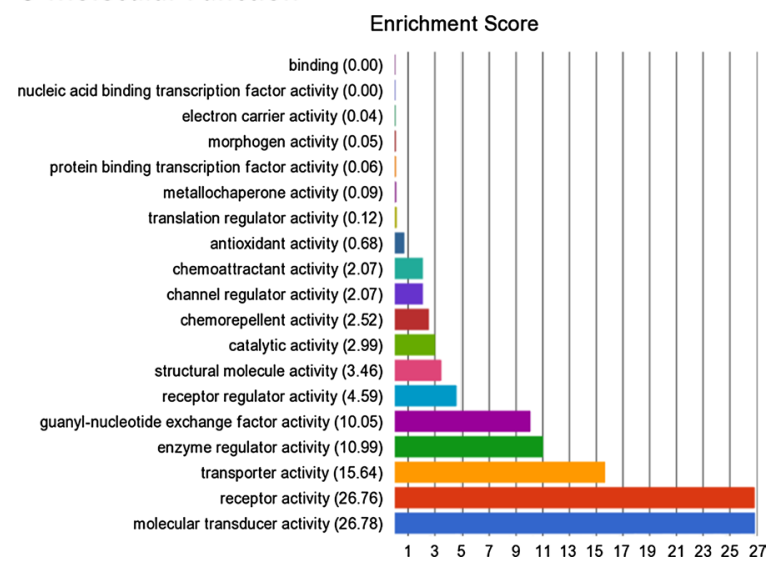

Fig. 2 GO enrichment results. a Biological processes, b cellular components, $\mathbf{c}$ molecular functions. These results are determined from the enriched genes which house $\geq 1$ top-ranked differentially methylated $\mathrm{CpG}$ site. The results refer to classes of genes in this population that are over represented and therefore may have an association with the disease phenotype compared to a control gene set

\section{Assessment of differential methylation between sample groups of the same origin}

Lastly, the methylation status was quantitatively determined between the two sample types within groups 1 and 2; the gDNA $(\mathrm{n}=8)$ samples in group 1 were directly compared to the gDNA samples $(n=8)$ in group 2 . This was then repeated for the clDNA ( $n=8$ vs. $n=8$ ) samples. Reassuringly, no CpG sites were significantly different between the two analysis groups.

\section{Discussion}

This study reports a comparison of the data generated by Illumina's Infinium MethylationEPIC BeadChip array technology for gDNA from peripheral blood leukocytes from 16 individuals and cIDNA, derived from EBV transformation of the same samples into cell lines performed by the ECACC. The methylationEPIC BeadChip array covers 862,927 CpG sites, which makes this the largest gDNA and clDNA methylation profiling study using this array [15].

DNA methylation plays a key role in epigenetic gene regulation and is the most well studied epigenetic factor [16]. It has been shown to alter with age and smoking status and therefore it was important to align the two analysis groups for age of T1D diagnosis and duration of diabetes [17-19].

Through this analysis, we have established that approximately $6 \%$ of the CpG sites covered by the MethylationEPIC array provided significantly different $p$-values between gDNA and clDNA based on their methylation beta values. We have not addressed potential causes of the observed differences in methylation [20]. This may be due to the method through which the cell-line transformation occurs [21-23]. One study assessing oral keratinocytes [24] has shown that EBV infection itself affects methylation levels, resulting in alterations to gene expression. Consistent with Sugawara and colleagues, they also demonstrated that the epigenetic alterations were retained following removal of the virus [24, 25].

Furthermore, the cell-line passage number has been demonstrated to affect epigenetic modifications. Grafodatskaya et al. [26] compared methylation patterns in blood cells with lymphoblastoid cell-lines (LCLs) of different passage numbers. They showed that low passage numbers and one freeze-thaw cycle does not affect methylation, but identified that LCLs can be prone to alterations in the DNA methylation at sporadic genomic locations when at high passage numbers.

Two genes AGXT and INS which have CpG sites included within the $6223 \mathrm{CpG}$ sites with a significant difference in methylation levels between gDNA vs. cIDNA (groups 1 and 2), had previously also been shown to be differentially methylated in an investigation which 
assessed 25,000 CpG sites from six individuals by Brennan and colleagues between gDNA and clDNA [21].

We have also shown that the CpG sites with differential methylation were not due to differences between the individuals within the two groups. Neither comparison, gDNA (group 1 vs. group 2), nor clDNA (group 1 vs. group 2) provided any significantly different levels of methylation.

We have previously shown that clDNA is a suitable replacement for gDNA in SNP-based analyses [27] and it is evident from these results, that the clDNA has potential to be an alternative material source for assessment of DNA methylation using the methylationEPIC array.

\section{Limitations}

A potential limitation is that the methylation data was generated for only 16 matched gDNA and clDNA samples, compared in groups of eight samples. It would be advantageous to repeat this on a larger sample size. As the cell line DNA was prepared off-site, it is possible that the number of freeze-thaw cycles of the two different collections could have been different, but we believe there were less than three freeze-thaw cycles for each aliquot.

\section{Supplementary information}

Supplementary information accompanies this paper at https://doi. org/10.1186/s13104-019-4853-4.

\section{Additional file 1: Appendix S1. Illustration of methods.}

Additional file 2: Table S1. Illumina Bead Array Controls Reporter for the gDNA and cIDNA samples. Table S2. Top-ranked differentially methylated CpG sites for gDNA vs. CIDNA (group 1). Table S3. Top-ranked differentially methylated CpG sites for gDNA vs. cIDNA (group 2). Table S4. Top-ranked $\mathrm{CpG}$ sites which differed between groups 1 and 2 (gDNA vs. CIDNA - ST2 and ST3). Table S5. Top-ranked CpG sites which were common between groups 1 and 2 (gDNA vs. CIDNA - ST2 and ST3). Table S6. GO enrichment analysis for genes where top-ranked $\mathrm{CpG}$ sites are located. Table S7. Enriched pathways (KEGG) for genes where top-ranked $\mathrm{CpG}$ sites are located.

Additional file 3: Figure S1. Illustration of the average methylation beta value patterns generated for each of the four groups. A) Group 1 (gDNA); B) Group 1 (cIDNA); C) Group 2 (gDNA); D) Group 2 (cIDNA).

\begin{abstract}
Abbreviations
BMI: body mass index; BP: blood pressure; BST: bisulphite treated; CKD: chronic kidney disease; CIDNA: cell-line derived DNA; CpG: cytosine-phosphate-guanine; DCs: diabetic controls; DKD: diabetic kidney disease; DNA: deoxyribose nucleic acid; EBV: Epstein-Barr virus; ECACC: European Collection of Authenticated Cell Cultures; eGFR: estimated glomerular filtration rate; ESRD: end-stage renal disease; gDNA: genomic DNA; GO: gene ontology; GoKinD: Genetics of Kidneys in Diabetes; KEGG: Kyoto Encyclopedia of Genes and Genomes; LCLs: lymphoblastoid cell-lines; $\mathrm{mmHg}$ : millimetres of mercury; QC: quality control; T1D: type 1 diabetes; T1DKD: type 1 diabetes and kidney disease; WGBS: whole-genome bisulphite sequencing.
\end{abstract}

\section{Acknowledgements}

We acknowledge the support of the funding bodies listed.

\section{Authors' contributions}

LSS, APM and AJM conceived the study and participated in its design and coordination. LJS and JK performed the analysis. LJS drafted the manuscript. All authors read and approved the final manuscript.

\section{Funding}

LSS is the recipient of a Northern Ireland Kidney Research Fund Fellowship (NIKRF). Funding was also provided by GEnetics of Nephropathy an International Effort (GENIE) and Science Foundation Ireland and the Department for the Economy, Northern Ireland (15/IA/3152). This work has been partly funded by the Medical Research Council (Award Reference MC_PC_15025) and the Public Health Agency R\&D Division (Award Reference STL/4760/13). None of the funding bodies had a role in the study design or analysis.

\section{Availability of data and materials}

The datasets generated and/or analysed during the current study are available from the corresponding author on reasonable request.

\section{Ethics approval and consent to participate}

Ethical approval for research involving the ECACC samples was granted by the Office for Research Ethics Committees Northern Ireland (ORECNI) — reference number ORECNI 175/23: RO321. Research ethics approval for the GoKinD samples was obtained from the South and West Multicentre Research Ethics Committee (MREC/98/6/71). All participants provided written informed consent for research. No individual level data is being reported in this manuscript.

\section{Consent for publication}

Not applicable.

\section{Competing interests}

The authors declare that they have no competing interests.

Received: 25 October 2019 Accepted: 10 December 2019

Published online: 21 December 2019

\section{References}

1. Smyth LI, McKay GJ, Maxwell AP, McKnight AJ. DNA hypermethylation and DNA hypomethylation is present at different loci in chronic kidney disease. Epigenetics. 2014;9(3):366-76.

2. Bansal A, Pinney SE. DNA methylation and its role in the pathogenesis of diabetes. Pediatr Diabetes. 2017:18(3):167-77.

3. Chu AY, Tin A, Schlosser P, Ko Y-A, Qiu C, Yao C, et al. Epigenome-wide association studies identify DNA methylation associated with kidney function. Nat Commun. 2017;8(1):1286.

4. Zhang H, Cai XU, Yi B, Huang J, Wang J, Sun J. Correlation of CTGF gene promoter methylation with CTGF expression in type 2 diabetes mellitus with or without nephropathy. Mol Med Rep. 2014;9(6):2138-44.

5. GuT, Falhammar H, Gu HF, Brismar K. Epigenetic analyses of the insulinlike growth factor binding protein 1 gene in type 1 diabetes and diabetic nephropathy. Clin Epigenetics. 2014;6(1):1-6.

6. Marumo T, Yagi S, Kawarazaki W, Nishimoto M, Ayuzawa N, Watanabe A, et al. Diabetes induces aberrant DNA methylation in the proximal tubules of the kidney. J Am Soc Nephrol. 2015;26(10):2388-97.

7. Ghattas M, El-shaarawy F, Mesbah N, Abo-Elmatty D. DNA methylation status of the methylenetetrahydrofolate reductase gene promoter in peripheral blood of end-stage renal disease patients. Mol Biol Rep. 2014;41(2):683-8.

8. Watanabe A, Marumo T, Kawarazaki W, Nishimoto M, Ayuzawa N, Ueda K, et al. Aberrant DNA methylation of pregnane $X$ receptor underlies metabolic gene alterations in the diabetic kidney. Am J Physiol Ren Physiol. 2018;314(4):F551-60

9. Nilsson E, Ling C. DNA methylation links genetics, fetal environment, and an unhealthy lifestyle to the development of type 2 diabetes. Clin Epigenetics. 2017;9(1):1-8. 
10. Dirks RAM, Stunnenberg HG, Marks H. Genome-wide epigenomic profiling for biomarker discovery. Clin Epigenetics. 2016. https://doi. org/10.1186/s13148-016-0284-4

11. Smyth LJ, Duffy S, Maxwell AP, McKnight AJ. Genetic and epigenetic factors influencing chronic kidney disease. Am J Physiol Renal Physiol. 2014;307(7):F757-76.

12. Teh AL, Pan H, Lin X, Lim Yl, Patro CPK, Cheong CY, et al. Comparison of Methyl-capture Sequencing vs. Infinium $450 \mathrm{~K}$ methylation array for methylome analysis in clinical samples. Epigenetics. 2016:11(1):36-48.

13. Pidsley R, Zotenko E, Peters TJ, Lawrence MG, Risbridger GP, Molloy $P$, et al. Critical evaluation of the Illumina MethylationEPIC BeadChip microarray for whole-genome DNA methylation profiling. Genome Biol. 2016:17(1):208.

14. Public Health England. Culture collections: about ECACC. https://www. phe-culturecollections.org.uk/collections/ecacc.aspx. Accessed 2 Aug 2018

15. Zhang C, Zhang R, Chen Z, Chen J, Ruan J, Lu Z, et al. Differential DNA methylation profiles of human B lymphocytes and Epstein-Barr virusimmortalized B lymphocytes. Chinese J Cancer Res. 2018;31(1):104-11.

16. Martin EM, Fry RC. Environmental Influences on the Epigenome: exposure- Associated DNA Methylation in Human Populations. Annu Rev Public Health. 2018;39:309-33.

17. López-otín C, Blasco MA, Partridge L, Serrano M, Kroemer G. The hallmarks of aging carlos. Cell. 2013;153(6):1194-217.

18. Klebaner D, Huang Y, Hui Q, Taylor JY, Goldberg J, Vaccarino V, et al. X chromosome-wide analysis identifies DNA methylation sites influenced by cigarette smoking. Clin Epigenetics. 2016;8:20

19. Fragou D, Pakkidi E, Aschner M, Samanidou V, Kovatsi L. Smoking and DNA methylation: correlation of methylation with smoking behavior and association with diseases and fetus development following prenatal exposure. Food Chem Toxicol. 2019;129(April):312-27.

20. Lam WKJ, Jiang P, Chan KCA, Peng W, Shang H, Heung MMS, et al. Methylation analysis of plasma DNA informs etiologies of Epstein-Barr virus-associated diseases. Nat Commun. 2019;10(1):1-11.
21. Brennan EP, Ehrich M, Brazil DP, Crean JK, Murphy M, Sadlier DM, et al. Comparative analysis of DNA methylation profiles in peripheral blood leukocytes versus lymphoblastoid cell lines. Epigenetics. 2009;4(3):159-64

22. Åberg K, Khachane AN, Rudolf G, Nerella S, Fugman DA, Tischfield JA, et al. Methylome-wide comparison of human genomic DNA extracted from whole blood and from EBV-transformed lymphocyte cell lines. Eur J Hum Genet. 2012;20(9):953-5.

23. Çalişkan M, Cusanovich DA, Ober C, Gilad Y. The effects of EBV transformation on gene expression levels and methylation profiles. Hum Mol Genet. 2011;20(8):1643-52.

24. Birdwell CE, Queen KJ, Kilgore PCSR, Rollyson P, Trutschl M, Cvek U, et al. Genome-wide DNA methylation as an epigenetic consequence of Epstein-Barr virus infection of immortalized keratinocytes. J Virol. 2014;88(19):11442-58.

25. Sugawara H, Iwamoto K, Bundo M, Ueda J, Ishigooka J, Kato T. Comprehensive DNA methylation analysis of human peripheral blood leukocytes and lymphoblastoid cell lines. Epigenetics. 2011;6(4):509-16.

26. Grafodatskaya D, Choufani S, Ferreira JC, Butcher DT, Lou Y, Zhao C, et al. EBV transformation and cell culturing destabilizes DNA methylation in human lymphoblastoid cell lines. Genomics. 2010;95(2):73-83.

27. Smyth LJ, Maxwell AP, Benson KA, Kilner J, McKay GJ, McKnight AJ. Validation of differentially methylated microRNAs identified from an epigenome-wide association study; Sanger and next generation sequencing approaches. BMC Res Notes. 2018;11:767.

\section{Publisher's Note}

Springer Nature remains neutral with regard to jurisdictional claims in published maps and institutional affiliations.
Ready to submit your research? Choose BMC and benefit from:

- fast, convenient online submission

- thorough peer review by experienced researchers in your field

- rapid publication on acceptance

- support for research data, including large and complex data types

- gold Open Access which fosters wider collaboration and increased citations

- maximum visibility for your research: over $100 \mathrm{M}$ website views per year

At BMC, research is always in progress.

Learn more biomedcentral.com/submissions 